\title{
ON THE $C^{*}$-ALGEBRA OF MATRIX-FINITE BOUNDED OPERATORS
}

\author{
V. MANUILOV
}

\begin{abstract}
Let $H$ be a separable Hilbert space with a fixed orthonormal basis. Let $\mathbb{B}^{(k)}(H)$ denote the set of operators, whose matrices have no more than $k$ non-zero entries in each line and in each column. The closure of the union (over $k \in \mathbb{N}$ ) of $\mathbb{B}^{(k)}(H)$ is a $C^{*}$-algebra. We study some properties of this $C^{*}$-algebra. We show that this $C^{*}$-algebra is not an $A W^{*}$-algebra, has a proper closed ideal greater than compact operators, and its group of invertibles is contractible.
\end{abstract}

\section{INTRODUCTION}

Hilbert spaces often come out with a fixed special orthonormal basis, e.g. $l^{2}(X)$ for a countable discrete space $X$, and the correspondence between operators and their matrices makes more sense. We got interested in the operators, whose matrices with respect to the fixed basis satisfy the following property: there is some $k \in \mathbb{N}$ such that each line and each column of the matrix has no more than $k$ non-zero entries. The norm closure of the union (over $k \in \mathbb{N}$ ) of these operators turns out to be a $C^{*}$-algebra, denoted by $\mathbb{B}_{f}(H)$. We establish some properties of this $C^{*}$-algebra, which is, in some aspects, similar to the algebra $\mathbb{B}(H)$ of all bounded operators on the Hilbert space $H$, e.g. the Kuiper theorem on contractibility of the group of invertibles holds also for invertibles in $\mathbb{B}_{f}(H)$. On the other hand, $\mathbb{B}_{f}(H)$ is not a von Neumann algebra, and even not an $A W^{*}$-algebra, and the quotient $\mathbb{B}_{f}(H) / \mathbb{K}(H)$ is not simple.

\section{Matrix-Finite Bounded operators on a Hilbert space}

Let $H$ be a separable Hilbert space with a fixed orthonormal basis $\left\{e_{n}\right\}_{n \in \mathbb{N}}$. For $k \in \mathbb{N}$, denote by $\mathbb{B}_{L}^{(k)}(H)$ (resp. $\mathbb{B}_{C}^{(k)}(H)$ ) the set of all bounded operators on $H$ such that each line (resp. each column) of their matrix (with respect to the fixed basis) contains no more than $k$ non-zero entries. Note that

$$
\mathbb{B}_{L}^{(k)}(H) \subset \mathbb{B}_{L}^{(l)}(H) \text { when } k<l \text {, and } \mathbb{B}_{C}^{(k)}(H)=\left(\mathbb{B}_{L}^{(k)}(H)\right)^{*} .
$$

Set also

$$
\mathbb{B}^{(k)}(H)=\mathbb{B}_{L}^{(k)}(H) \cap \mathbb{B}_{C}^{(k)}(H) .
$$

Let $a, b \in \mathbb{B}(H), A=\left(a_{i j}\right), B=\left(b_{i j}\right)$ their matrices.

Lemma 1.1. If $a, b \in \mathbb{B}_{L}^{(k)}(H)$ then $a+b \in \mathbb{B}_{L}^{(2 k)}(H)$.

Proof. Obvious.

Lemma 1.2. If $a, b \in \mathbb{B}_{L}^{(k)}(H)$ then $a b \in \mathbb{B}_{L}^{\left(k^{2}\right)}(H)$.

Proof. Let $c_{i l}=\sum_{j \in \mathbb{N}} a_{i j} b_{j l}$. Fix $i$. There exist $j_{1}, \ldots, j_{k} \in \mathbb{N}$ such that $a_{i j}=0$ if $j \notin\left\{j_{1}, \ldots, j_{k}\right\}$. For each $j_{m}, m=1, \ldots, k$, there exist $l_{1}^{(m)}, \ldots, l_{k}^{(m)} \in \mathbb{N}$ such that $b_{j_{m} l}=0$ if $l \notin\left\{l_{1}^{(m)}, \ldots, l_{k}^{(m)}\right\}$. So $c_{i l}=0$ for $l \notin\left\{l_{n}^{(m)}\right\}_{n, m=1}^{k}$, hence the $i$-th line contains no more than $k^{2}$ non-zero entries. 
Set $\mathbb{B}^{(\infty)}(H)=\cup_{k \in \mathbb{N}} \mathbb{B}^{(k)}(H)$. This algebra was defined and studied in [5], AbadieCortinas.

Corollary 1.3. $\mathbb{B}^{(\infty)}(H)$ is a unital $*$-subalgebra in $\mathbb{B}(H)$.

Note that the correspondence between operators and their matrices is more clear for operators in $\mathbb{B}^{(\infty)}(H)$.

Lemma 1.4. Let $\left(a_{i j}\right)_{i, j \in \mathbb{N}}$ be a matrix such that each line and each column contains no more than $k$ non-zero entries, and there exists $C$ such that $\left|a_{i j}\right|<C$ for any $i, j \in \mathbb{N}$. Then the operator a given by this matrix is bounded.

Proof. Let the non-zero elements in the $i$-th line are $a_{i, j_{1}^{(i)}}, \ldots, a_{i, j_{k}^{(i)}}$. Define matrices $a^{(1)}, \ldots, a^{(k)}$ by

$$
\left(a^{(m)}\right)_{i k}=\left\{\begin{array}{cl}
a_{i, j_{m}^{(i)}} & \text { if } k=j_{m}^{(i)} \\
0 & \text { if } k \neq j_{m}^{(i)} .
\end{array}\right.
$$

Then each matrix $a^{(m)}$ has a single non-zero element in each line, and $a=a^{(1)}+\cdots+a^{(k)}$.

Let $\xi=\left(\xi_{n}\right)_{n \in \mathbb{N}} \in H$. Then $a^{(m)} \xi=\left(a_{1, j_{m}^{(1)}} \xi_{j_{m}^{(1)}}, a_{2, j_{m}^{(2)}} \xi_{j_{m}^{(2)}}, \ldots\right)$, hence

$$
\left\|a^{(m)} \xi\right\|^{2}=\left|a_{1, j_{m}^{(1)}} \xi_{j_{m}^{(1)}}\right|^{2}+\left|a_{2, j_{m}^{(2)}} \xi_{j_{m}^{(2)}}\right|^{2}+\cdots<C^{2}\left(\left|\xi_{j_{m}^{(1)}}\right|^{2}+\left|\xi_{j_{m}^{(2)}}\right|^{2}+\cdots\right) \leq C^{2} k \sum_{j \in \mathbb{N}}\left|\xi_{j}\right|^{2}
$$

as each $j$ appears not more than $k$ times among the numbers $j_{m}^{(1)}, j_{m}^{(2)}, \ldots$

Thus $\left\|a^{(m)}\right\|^{2}<C^{2} k$. As $\|a\| \leq\left\|a^{(1)}\right\|+\cdots+\left\|a^{(k)}\right\|$, we get $\|a\|<C k^{3 / 2}$.

Denote by $\mathbb{B}_{f}(H)$ the norm closure of $\mathbb{B}^{(\infty)}(H)$. Then it is a $C^{*}$-algebra.

Lemma 1.5. $M_{m}\left(\mathbb{B}_{f}(H)\right) \cong \mathbb{B}_{f}(H)$ for any $m \in \mathbb{N}$.

Proof. Obvious.

Let $\mathbb{K}(H)$ denote the $C^{*}$-algebra of compact operators on $H$.

Lemma 1.6. $\mathbb{K}(H) \subset \mathbb{B}_{f}(H)$.

Proof. Any compact operator can be approximated by operators with only finite number of non-zero entries.

\section{Difference from $\mathbb{B}(H)$}

The following examples show that the $C^{*}$-algebra $\mathbb{B}_{f}(H)$ is smaller than $\mathbb{B}(H)$, but not too much smaller.

Proposition 2.1. Define the vectors $a_{n} \in H, n \in \mathbb{N}$, by

$$
a_{1}=(1,0,0, \ldots) ; a_{2}=\left(0, \frac{1}{\sqrt{2}}, \frac{1}{\sqrt{2}}, 0,0, \ldots\right) ; a_{3}=\left(0,0,0, \frac{1}{\sqrt{3}}, \frac{1}{\sqrt{3}}, \frac{1}{\sqrt{3}}, 0,0, \ldots\right) ; \ldots
$$

Define an isometry $v$ by $v\left(e_{n}\right)=a_{n}$. Then $v \notin \mathbb{B}_{f}(H)$.

Proof. If $v \in \mathbb{B}_{f}(H)$ then for any $\varepsilon>0$ there exists $k \in \mathbb{N}$ and $b \in \mathbb{B}_{f}^{(k)}(H)$ such that $\|v-b\|<\varepsilon$. In particular, $\left\|(v-b) e_{n}\right\|<\varepsilon$ for any $n \in \mathbb{N}$. The vector $b e_{n}$ has no more than $k$ non-zero coordinates, hence $\left\|a_{n}-b e_{n}\right\|^{2} \geq \frac{n-k}{n}$. Taking $\varepsilon<\frac{1}{2}$ and $n>2 k$, we get a contradiction. 
The same argument can be used in the next example:

Proposition 2.2. Let $L \subset H$ be the closed subspace spanned by $a_{1}, a_{2}, \ldots$, and let $b_{1}, b_{2}, \ldots$ be an orthonormal basis for $L^{\perp}$. Set $u\left(e_{2 n-1}\right)=a_{n}, u\left(e_{2 n}\right)=b_{n}$. Then $u$ is a unitary, and $u \notin \mathbb{B}_{f}(H)$.

Proposition 2.3. Let $H=\oplus_{n \in \mathbb{N}} \mathbb{C}^{n}$ with the basis consisting of the standard bases of $\mathbb{C}^{n}$, $n \in \mathbb{N}$. Let $p_{n}=\left(\begin{array}{ccc}\frac{1}{n} & \cdots & \frac{1}{n} \\ \vdots & \ddots & \vdots \\ \frac{1}{n} & \cdots & \frac{1}{n}\end{array}\right)$ denote the projection onto the line $(x, \ldots, x), x \in \mathbb{C}$, in $\mathbb{C}^{n}$. Set $p=\oplus_{n \in \mathbb{N}} p_{n}$. Then $p \in \mathbb{B}_{f}(H)$. Note that we have $p=$ vv $v^{*}$, where $v \in \mathbb{B}(H) \backslash \mathbb{B}_{f}(H)$ is the isometry from Proposition 2.1.

Proof. The proof uses expander graphs [8] and essentially is contained in [6], where it is shown that the direct sum of one-dimensional projections $\oplus_{n \in \mathbb{N}} p_{m_{n}}$ lies in the uniform Roe algebra (hence in $\mathbb{B}_{f}(H)$, cf. Lemma 5.3 below) for certain increasing sequences $\left\{m_{n}\right\}$ of sizes. We only add a remark that one may take $m_{n}=n$.

Let $X=(V, E)$ be a $d$-regular graph, without loops and multiple edges, with the set $V$ of vertices and the set $E$ of edges. Let $|V|=m$. The $m$-dimensional Hilbert space $l^{2}(V)$ is endowed with the standard basis consisting of characteristic functions of the vertices. The Laplacian on $l^{2}(V)$ is the positive operator $\mathcal{L}$ given by the matrix $\left(L_{v, w}\right)_{v, w \in V}$, where

$$
L_{v, w}=\left\{\begin{aligned}
1, & \text { if } v=w \\
-\frac{1}{d}, & \text { if } v, w \text { are adjacent } \\
0, & \text { otherwise }
\end{aligned}\right.
$$

It is known that $\|\mathcal{L}\| \leq 2$ for any $m \in \mathbb{N}$. It is also easy to see that the matrix $L$ has no more than $d+1$ non-zero entries in each line and in each column for any $m \in \mathbb{N}$.

The smallest eigenvalue $\lambda_{0}$ of $\mathcal{L}$ is zero, and the corresponding eigenfunctions are constants, so the projection onto the kernel of $\mathcal{L}$ is the spectral projection onto this eigenspace, and its matrix equals $p_{m}$.

If $d \geq 5$ then for each $n \in \mathbb{N}$ there exists a $\frac{1}{2}$-expander graph $X_{n}=\left(V_{n}, E_{n}\right)$ with $\left|V_{n}\right|=2 n$ ([8], Proposition 1.2.1), and it follows ([8], Section 4.2) that the second smallest eigenvalue $\lambda_{1}$ of each Laplacian $\mathcal{L}_{n}$ of $X_{n}$ satisfies $\lambda_{1} \geq \delta$ for some $\delta>0$.

Let $\left\{f_{s}\right\}_{s \in \mathbb{N}}$ be a sequence of polynomials such that

- $f_{s}(0)=1$ for any $s \in \mathbb{N}$;

- $\left|f_{s}(t)\right| \leq \frac{1}{s}$ for any $t \in[\delta, 2]$.

Set $H=\oplus_{n \in \mathbb{N}} l^{2}\left(V_{n}\right)$ with the basis obtained by uniting the bases of each $l^{2}\left(V_{n}\right)$, and let $\mathcal{L}=\oplus_{n \in \mathbb{N}} \mathcal{L}_{n}$.

Note that each $\mathcal{L}_{n}$ has no more than $d+1$ non-zero entries in each line and in each column. By Lemma 1.1 and Lemma 1.2, we conclude that for any $s \in \mathbb{N}$ there exists $k=k(s) \in \mathbb{N}$ such that each line and each column of $f_{s}\left(\mathcal{L}_{n}\right)$ contains no more than $k$ non-zero entries. Therefore, $f_{s}(\mathcal{L})=\oplus_{n \in \mathbb{N}} \mathcal{L}_{n} \in \mathbb{B}^{(k)}(H)$. As the spectrum of $\mathcal{L}$ lies in $\{0\} \cup[\delta, 2]$, the operators $f_{s}(\mathcal{L})$ converge to the spectral projection $p^{\prime}=\oplus_{n \in \mathbb{N}} p_{2 n}$ onto the kernel of $\mathcal{L}$ :

$$
\lim _{s \rightarrow \infty}\left\|f_{s}(\mathcal{L})-p^{\prime}\right\|=0
$$

hence $p^{\prime} \in \mathbb{B}_{f}(H)$.

Similarly, $p^{\prime \prime}=p_{1} \oplus\left(\oplus_{n \in \mathbb{N}}\left(p_{2 n} \oplus p_{2 n} \oplus 0\right)\right) \in \mathbb{B}_{f}(H)$. Note that

$$
\left\|p_{2 n+1}-\left(\begin{array}{cc}
p_{2 n} & 0 \\
0 & 0
\end{array}\right)\right\|=\left\|\frac{1}{2 n+1}\left(\begin{array}{cc}
-p_{2 n} & l \\
l^{*} & 1
\end{array}\right)\right\| \leq \frac{1}{2 n+1}\left(\left\|p_{2 n}\right\|+2\|l\|+1\right)=\frac{2+2 \sqrt{2 n}}{2 n+1},
$$


where $l$ is the column of $2 n$ units. So, $p-p^{\prime \prime} \in \mathbb{K}(H)$, and, by Lemma 1.6, $p \in \mathbb{B}_{f}(H)$.

Note that, by spectral theorem, any selfadjoint $a \in \mathbb{B}(H)$ can be approximated by linear combinations of projections, so if all projections in $H$ would lie in $\mathbb{B}_{f}(H)$ then $a \in \mathbb{B}_{f}(H)$, hence any $b \in \mathbb{B}(H)$ must lie in $\mathbb{B}_{f}(H)$, which is false. Therefore, there exists a projection in $\mathbb{B}(H) \backslash \mathbb{B}_{f}(H)$. Here is an explicit example.

Proposition 2.4. Let $u$ be the unitary from Proposition 2.2 on a Hilbert space $H$, and let $p=\frac{1}{2}\left(\begin{array}{cc}1 & u \\ u^{*} & 1\end{array}\right)$ be a projection in $H \oplus H$ (with the basis obtained by uniting bases of the two copies of $H)$. Then $p \notin \mathbb{B}_{f}(H \oplus H)$.

Proof. This follows from Lemma 1.5 and from $u \notin \mathbb{B}_{f}(H)$.

\section{IDEALS}

It is known that $\mathbb{K}(H)$ is the unique closed two-sided ideal in $\mathbb{B}(H)$. This is not true for $\mathbb{B}_{f}(H)$.

Let $a \in \mathbb{B}_{f}(H),\left(a_{i j}\right)_{i, j \in \mathbb{N}}$ the matrix of $a$ (recall that the basis is fixed). Set

$$
\mathbb{I}(H)=\left\{a \in \mathbb{B}_{f}(H): \lim _{n \rightarrow \infty} \sup _{i, j \geq n}\left|a_{i j}\right|=0\right\} .
$$

Obviously, $\mathbb{K}(H) \subset \mathbb{I}(H)$. It is easy to see that the projection $p$ from Proposition 2.3 lies in $\mathbb{I}(H)$ and is not compact, hence $\mathbb{I}(H)$ is strictly bigger than $\mathbb{K}(H)$.

A similar ideal in uniform Roe algebras was studied, e.g. in [11], under the name of the ideal of ghost operators.

Theorem 3.1. $\mathbb{I}(H)$ is the maximal closed two-sided proper ideal in $\mathbb{B}_{f}(H)$.

Proof. First, note that $\mathbb{I}(H)$ is closed. Indeed, suppose that $\left(a^{(\iota)}\right)_{\iota \in I}$ converges to $a \in$ $\mathbb{B}_{f}(H)$, and $a^{(\iota)} \in \mathbb{I}(H)$ for any $\iota \in I$. Fix $\varepsilon>0$, then there is $\kappa \in I$ such that $\left\|a^{(\iota)}-a\right\|<\varepsilon$ for any $\iota \geq \kappa$. Therefore, $\left|a_{i j}^{(\iota)}-a_{i j}\right|<\varepsilon$ for any $i, j \in \mathbb{N}$. Then

$$
\left|a_{i j}\right| \leq\left|a_{i j}^{(\kappa)}\right|+\left|a_{i j}-a_{i j}^{(\kappa)}\right|<\left|a_{i j}^{(\kappa)}\right|+\varepsilon .
$$

As $a^{(\kappa)} \in \mathbb{I}(H)$, there exists $N$ such that $\left|a_{i j}^{(\kappa)}\right|<\varepsilon$ for any $i, j>N$. Then $\left|a_{i j}\right|<2 \varepsilon$ for any $i, j>N$.

Now let $a \in \mathbb{I}(H), b \in \mathbb{B}^{(k)}(H)$ for some $k$. Let us check that $a b \in \mathbb{I}(H)$. Due to the involution, this would imply $b a \in \mathbb{I}(H)$. Set $c_{i l}=\sum_{j \in \mathbb{N}} a_{i j} b_{j l}$. Take $\varepsilon>0$ and find $N$ such that $\left|a_{i j}\right|<\varepsilon$ for $i, j>N$. After fixing $N$, we can find $M$ such that $b_{j l}=0$ for any $j \leq N$ and any $l>M$ (see Lemma 1.2). Then, for $i>N$ and $l>M$ we have

$$
\left|c_{i l}\right|=\left|\sum_{j \in \mathbb{N}} a_{i j} b_{j l}\right|=\left|\sum_{j>N} a_{i j} b_{j l}\right|<\varepsilon k\|b\|
$$

(we use here that the sum contains not more than $k$ non-zero summands and that $\left|b_{j l}\right| \leq$ $\|b\|$ for all $j, l \in \mathbb{N})$. Thus $\mathbb{I}(H)$ is a two-sided ideal in $\mathbb{B}_{f}(H)$.

It remains to show that $\mathbb{I}(H)$ is the maximal proper closed two-sided ideal in $\mathbb{B}_{f}(H)$. Assume that there exists an ideal $\mathbb{J}$ and a selfadjoint $a \in \mathbb{J} \backslash \mathbb{I}(H)$. We are going to construct a subspace $L \subset H$ and an isometry $u: H \rightarrow L$ in two different cases. First, consider the case when there exists a sequence $\left\{i_{n}\right\}_{n \in \mathbb{N}}$ such that $\left\|a_{i_{n}, i_{n}}\right\|>\delta$ for some $\delta>0$. As $\left.\mathbb{B}^{(} \infty\right)(H)$ is dense in $\mathbb{B}_{f}(H)$, there exists $k \in \mathbb{N}$ and a selfadjoint $a^{(k)} \in \mathbb{B}^{(k)}(H)$ 
such that $\left\|a-a^{(k)}\right\|<\delta / 4$. Then $\left|a_{i_{n}, i_{n}}^{(k)}\right|>3 \delta / 4$. Let us pass to a subsequence $\left\{i_{n_{m}}\right\}_{m \in \mathbb{N}}$ inductively: set $n_{1}=1$, and if $m_{n}$ is already fixed then let $n_{m+1}$ be defined by the condition

$$
i_{n_{m+1}}>l \text { for any } l \in \mathbb{N} \text { such that at least one of } a_{i_{n_{1}}, l}^{(k)}, \ldots, a_{i_{n_{m}}, l}^{(k)} \text { is non-zero }
$$

(for each $n$, there is not more than $k$ non-zero entries $a_{i_{n}, j}^{(k)}$ ). Then let $L \subset H$ be a closed subspace spanned by the vectors $e_{i_{m}}, m \in \mathbb{N}$, and let $p_{L} \in \mathbb{B}_{f}(H)$ be the projection onto $L$. By (1), $\left.p_{L} a^{(k)}\right|_{L}$ is a diagonal operator:

$$
a_{i_{n_{m}}, i_{n_{m^{\prime}}}}^{(k)}=0 \text { if } m \neq m^{\prime}, \quad \text { and } \quad\left|a_{i_{n_{m}}, i_{n_{m}}}^{(k)}\right|>3 \delta / 4 .
$$

Then $\left\|p_{L} a^{(k)} \xi\right\|>\frac{3 \delta}{4}\|\xi\|$ and

$$
\left\|p_{L} a \xi\right\| \geq\left\|p_{L} a^{(k)} \xi\right\|-\left\|p_{L}\left(a^{(k)}-a\right) \xi\right\|>\frac{3 \delta}{4}\|\xi\|-\frac{\delta}{4}\|\xi\|=\frac{\delta}{2}\|\xi\|
$$

for any $\xi \in L$. Let $u: H \rightarrow L$ be the isometry defined by $u\left(e_{m}\right)=e_{i_{n_{m}}}$. Then $u \in \mathbb{B}_{f}(H)$.

Otherwise, assume that $\lim _{i \rightarrow \infty} a_{i i}=0$. As $a \notin \mathbb{I}(H)$, there exists some $\delta>0$ and sequences $\left\{i_{n}\right\},\left\{j_{n}\right\}_{n \in \mathbb{N}}$ such that $i_{n+1}, j_{n+1}>\max \left(i_{n}, j_{n}\right), j_{n} \neq i_{n}$, and $\left|a_{i_{n}, j_{n}}\right|>\delta$ for any $n \in \mathbb{N}$. As in the first case, find a selfadjoint $a^{(k)} \in \mathbb{B}^{(k)}(H)$ such that $\left\|a-a^{(k)}\right\|<\delta / 6$. Then in the two-by-two matrices

$$
\left(\begin{array}{ll}
a_{i_{n}, i_{n}}^{(k)} & a_{i_{n}, j_{n}}^{(k)} \\
a_{j_{n}, i_{n}}^{(k)} & a_{j_{n}, j_{n}}^{(k)}
\end{array}\right)
$$

the diagonal entries satisfy $\left|a_{i, i}^{(k)}\right|<\delta / 6$ for sufficiently great $n$, while the off-diagonal entries have modulus greater than $5 \delta / 6$, hence there exists some $n_{0} \in \mathbb{N}$ such that the matrices (2) are bounded from below by $2 \delta / 3$ for $n>n_{0}$.

Once again let us pass inductively to a subsequence $\left\{n_{m}\right\}_{m \in \mathbb{N}}$. Set $n_{1} \geq n_{0}$, and if $n_{m}$ is already fixed then let $n_{m+1}$ be defined by the condition

$$
\min \left(i_{n_{m+1}}, j_{n_{m+1}}\right)>l
$$

for any $l \in \mathbb{N}$ such that at least one of $a_{i_{n_{1}}, l}^{(k)}, \ldots, a_{i_{n_{m}}, l}^{(k)}, a_{j_{n_{1}}, l}^{(k)}, \ldots, a_{j_{n_{m}}, l}^{(k)}$ is non-zero.

Let $L$ be a closed subspace spanned by $e_{i_{n_{m}}}, e_{j_{n_{m}}}, m \in \mathbb{N}$. Then $p_{L} a^{(k)}$ is a blockdiagonal operator with two-dimensional blocks of the form (2), hence $\left\|p_{L} a^{(k)} \xi\right\|>\frac{2 \delta}{3}$ for any $\xi \in L$. Then

$$
\left\|p_{L} a \xi\right\| \geq\left\|p_{L} a^{(k)} \xi\right\|-\left\|p_{L}\left(a^{(k)}-a\right) \xi\right\|>\frac{2 \delta}{3}\|\xi\|-\frac{\delta}{6}\|\xi\|=\frac{\delta}{2}\|\xi\|
$$

for any $\xi \in L$. Similarly to the first case, let $u: H \rightarrow L$ be the isometry defined by $u\left(e_{2 m-1}\right)=e_{i_{m}}, u\left(e_{2 m}\right)=e_{j_{n_{m}}}$. Again we have $u \in \mathbb{B}_{f}(H)$.

The further argument is standard [4]. In both cases, for any $\xi \in H$ we have $\left\|u^{*} p_{L} a u \xi\right\|>$ $\frac{\delta}{2}\|\xi\|$ for any $\xi \in H$, hence $u^{*} p_{L} a u$ is invertible, therefore, $1 \in \mathbb{J}$ and $\mathbb{J}=\mathbb{B}_{f}(H)$.

\section{Similar $C^{*}$-AlgEBraS}

We know three $C^{*}$-algebras, which seem similar to $\mathbb{B}_{f}(H)$. We don't know if they all are the same or different, but it is easy to see that $\mathbb{B}_{f}(H)$ lies in all of them.

In [12] the following $C^{*}$-subalgebra of $\mathbb{B}(H)$ was defined. Let $a \in \mathbb{B}(H),\left(a_{i j}\right)_{i, j \in \mathbb{N}}$ its matrix. The operator $a$ is called $l_{1}$-bounded if there is $m \in(0, \infty)$ such that $\sum_{i=1}^{\infty}\left|a_{i j}\right|<m$ for any $j \in \mathbb{N}$ and $\sum_{j=1}^{\infty}\left|a_{i j}\right|<m$ for any $i \in \mathbb{N}$. It is shown in [12] that $l_{1}$-bounded 
operators form a *-algebra, and its norm-closure is a $C^{*}$-algebra. Let us denote it by $\mathbb{B}_{T}(H)$.

Lemma 4.1. $\mathbb{B}_{F}(H) \subset \mathbb{B}_{T}(H)$.

Proof. Let $a \in \mathbb{B}^{(k)}(H)$. Then $\sum_{j=1}^{\infty}\left|a_{i j}\right| \leq k\|a\|$.

Another $C^{*}$-subalgebra of $\mathbb{B}(H)$ can be defined as follows.

Let $\omega$ be a free ultrafilter on $\mathbb{N}$. Set

$$
\begin{gathered}
\mathcal{H}=\left\{\xi=\left(\xi_{n}\right)_{n \in \mathbb{N}}: \sup _{n}\left\|\xi_{n}\right\|<\infty\right\}, \\
\langle\xi, \eta\rangle=\lim _{\omega}\left\langle\xi_{n}, \eta_{n}\right\rangle, \quad \xi, \eta \in \mathcal{H}, \\
\mathcal{H}_{0}=\{\xi \in \mathcal{H}:\langle\xi, \xi\rangle=0\}, \quad H^{\omega}=\mathcal{H} / \mathcal{H}_{0} .
\end{gathered}
$$

Then $H^{\omega}$ is a (non-separable) Hilbert space, and $\mathbb{B}(H)$ is obviously represented on $H^{\omega}$ by

$$
\pi(a) \xi=\left(a \xi_{n}\right)_{n \in \mathbb{N}}, \quad \pi: \mathbb{B}(H) \rightarrow \mathbb{B}\left(H^{\omega}\right) .
$$

As $H^{\omega}$ contains a copy of $H$ consisting of constant sequences, $\pi$ is injective.

For $k \in \mathbb{N}$ let $\mathcal{H}^{(k)}$ denote the set of all sequences $\xi \in \mathcal{H}$ such that not more than $k$ entries are non-zero. Although each $\mathcal{H}^{(k)}$ is not a linear subspace, their union

$$
\mathcal{H}^{(\infty)}=\cup_{k \in \mathbb{N}} \mathcal{H}^{(k)}
$$

is. Then $\mathcal{H}^{(\infty)} / \mathcal{H}_{0}$ is a linear subspace of $H^{\omega}$. Denote its closure by $H_{f}^{\omega}$. It is a proper subspace of $H^{\omega}$.

Lemma 4.2. Let $a \in \mathbb{B}(H)$. If $a \in \mathbb{B}_{f}(H)$ then $H_{f}^{\omega}$ is invariant for $\pi(a)$.

Proof. Let $a \in \mathbb{B}^{(k)}(H), \xi=\left(\xi_{n}\right)_{n \in \mathbb{N}} \in \mathcal{H}^{(l)}$. Then $a \xi_{n}$ has not more than $k l$ non-zero coordinates, hence $\left(a \xi_{n}\right)_{n \in \mathbb{N}} \in \mathcal{H}^{(k l)}$, hence $\pi(a)$ maps $\mathcal{H}^{(k)}+\mathcal{H}_{0}$ to $\mathcal{H}^{(k l)}+\mathcal{H}_{0}$, thus leaves $\mathcal{H}^{(\infty)} / \mathcal{H}_{0}$ invariant.

Set $\mathbb{B}_{\omega}(H)=\left\{a \in \mathbb{B}(H): \pi(a)\left(H_{f}^{\omega}\right) \subset H_{f}^{\omega}\right\}$. It is a $C^{*}$-algebra, and $\mathbb{B}_{f}(H) \subset \mathbb{B}_{\omega}(H) \subset$ $\mathbb{B}(H)$.

One more $C^{*}$-algebra is the multiplier $C^{*}$-algebra $M(\mathbb{I}(H))$ of the ideal $\mathbb{I}(H)$. As $\mathbb{K}(H) \subset \mathbb{I}(H) \subset \mathbb{B}(H)$, we have $M(\mathbb{I}(H)) \subset \mathbb{B}(H)$.

It is easy to see that the isometry from Proposition 2.1 lies in neither of the three $C *$-algebras.

Problem 4.3. Are the $C^{*}$-algebras $\mathbb{B}_{f}(H), \mathbb{B}_{T}(H), \mathbb{B}_{\omega}(H), M(\mathbb{I}(H))$ different?

\section{EMBEDDINGS}

We do not know if any separable $C^{*}$-algebra $A$ admits an injective $*$-homomorphism into $\mathbb{B}_{f}(H)$, but here are four easy cases when the answer is positive.

Lemma 5.1. Let a discrete group $\Gamma$ act on a countable discrete space $X$. Then the reduced crossed product $C_{b}(X) \rtimes_{r} \Gamma$ by the algebra $C_{b}(X)$ of bounded functions on $\Gamma$ admits an injective $*$-homomorphism into $\mathbb{B}_{f}(H)$. In particular, the reduced group $C^{*}$-algebra $C_{r}^{*}(\Gamma)$ admits an injective $*$-homomorphism into $\mathbb{B}_{f}(H)$. 
Proof. Set $H=l^{2}(X)$ with the basis of the characteristic functions $\delta_{x}$ of individual points $x \in X$. Let $a \in \mathbb{C}[\Gamma], a=\lambda_{1} g_{1}+\ldots+\lambda_{r} g_{r}$, be a finite linear combination of group elements $g_{1}, \ldots, g_{r} \in \Gamma, \lambda_{1}, \ldots, \lambda_{r} \in \mathbb{C}$. As $g \delta_{x}=\delta_{g x}, g^{-1} \delta_{x}=\delta_{g^{-1} x}$, the matrix of the operator on $l^{2}(\Gamma)$ given by $a$ has no more than $r$ non-zero entries in each line and in each column. This proves inclusion of $C_{r}^{*}(\Gamma)$ in $\mathbb{B}_{f}(H)$. Operators of multiplication by functions on $X$ are diagonal, hence trivially lie in $\mathbb{B}_{f}(H)$. The case $X=\Gamma$ proves Lemma for $C_{r}^{*}(\Gamma)$.

Lemma 5.2. Let $\mathbb{F}_{n}$ be a free group on $n$ generators. Then the full group $C^{*}$-algebra $C^{*}\left(\mathbb{F}_{n}\right)$ admits an injective $*$-homomorphism into $\mathbb{B}_{f}(H)$.

Proof. It is shown in [8], Proposition 2.3, that the universal norm on $\mathbb{C}\left[\mathbb{F}_{n}\right]$ equals the supremum of the norms of representations of $\mathbb{F}_{n}$ which factor through a finite quotient. By Lemma 5.1, $C^{*}\left(\mathbb{F}_{n}\right)$ embeds into $\mathbb{B}_{f}(H)$.

Let $X$ be a metric space of bounded geometry, which means that, for any $R>0$, the number of points in each ball of raduis $R$ is uniformly bounded. The uniform Roe algebra $C^{*}(X)$ [10] is generated on $H=l^{2}(X)$ by operators $a \in \mathbb{B}(H)$, whose matrices satisfy $a_{x y}=0$ if $d(x, y)>R, R>0$ (here $x, y \in X, a_{x y}=\left\langle\delta_{x}, a \delta_{y}\right)$.

Lemma 5.3. The uniform Roe algebra $C^{*}(X)$ for metric spaces of bounded geometry admits an injective $*$-homomorphism into $\mathbb{B}_{f}(H)$.

Proof. Obvious.

Let $V=\mathbb{N}$, and let $G=(V, E)$ be an infinite, uniformly locally finite (ULF) graph, i.e. $E$ denotes the set of edges of $G$, two vertices $v, w \in V$ are adjacent $(v \sim w)$ if there is an edge $e \in E$ that connects $v$ and $w$, each vertex $v$ has a finite number $\operatorname{deg} v$ of adjacent vertices, and these numbers $\operatorname{deg} v$ are uniformly bounded on $V$. Let $A_{G}=\left(\left(a_{G}\right)_{v, w}\right)_{v, w \in V}$ be the adjacency matrix, i.e. the infinite matrix defined by $\left(a_{G}\right)_{v, w}= \begin{cases}1, & \text { if } v \sim w ; \\ 0, & \text { otherwise. }\end{cases}$ Each line and each column of the matrix $A_{G}$ has no more than $k$ non-zero entries for some $k \in \mathbb{N}$. By Lemma 1.4, this matrix determines a bounded operator $a_{G}$, called adjacency operator. Let $B(G)=C^{*}\left(l^{\infty}(V), a_{G}\right)$ be the $C^{*}$-subalgebra of $\mathbb{B}\left(l^{2}(V)\right)$ generated by (operators of multiplication by) $l^{\infty}(V)$ and by $a_{G}$.

Lemma 5.4. The $C^{*}$-algebra $B(G)$ defined above admits an injective *-homomorphism into $\mathbb{B}_{f}(H)$.

Proof. Obvious.

Problem 5.5. Are there separable $C^{*}$-algebras that do not admit an injective *homomorphism into $\mathbb{B}_{f}(H)$ ?

\section{Contractibility of the Group of invertibles}

Theorem 6.1. The group $G L_{f}(H)$ of invertible elements of $\mathbb{B}_{f}(H)$ is contractible.

Proof. The proof follows the original proof of Kuiper [7]. Let $f: \mathbb{S}^{n} \rightarrow G L_{f}(H)$ be a continuous map from a sphere. A preliminary step is to deform $f$ to a map $f_{1}$ such that $f_{1}\left(\mathbb{S}^{n}\right)$ is contained in the linear span of some $g_{1}, \ldots, g_{N} \in G L_{f}(H)$. After a small 
deformation, we may assume that there exists $k \in \mathbb{N}$ such that $g_{j} \in G L^{(k)}(H), j=$ $1, \ldots, N$, where $G L^{(k)}(H)=G L_{f}(H) \cap \mathbb{B}^{(k)}(H)$.

Then we have to find a sequence of unit vectors $a_{i}, a_{i}^{\prime}$, and of finitedimensional subspaces $A_{i} \subset H$ with the following properties:

- $A_{i}=\operatorname{Span}\left(a_{i}, g_{1}\left(a_{i}\right), \ldots, g_{N}\left(a_{i}\right), a_{i}^{\prime}\right)$;

- $a_{i}^{\prime} \perp a_{i}, g_{1}\left(a_{i}\right), \ldots, g_{N}\left(a_{i}\right)$, and $a_{i}^{\prime} \perp A_{l}$ for $l<i$;

- $a_{i} \in \cap_{l=1}^{i-1}\left[A_{l}^{\perp} \cap\left(\cap_{j=1}^{N} g_{j}^{-1}\left(A_{l}^{\perp}\right)\right)\right]$.

We claim that if $g_{j} \in G L^{(k)}(H)$ then the vectors $a_{i}, a_{i}^{\prime}, i \in \mathbb{N}$, can be taken from the basis $\left\{e_{n}\right\}_{n \in \mathbb{N}}$ of $H$.

Indeed, one can start with $a_{1}=e_{1}$. As $g_{j} \in G L^{(k)}(H)$, there exists $r_{1}$ such that $a_{1}, g_{1}\left(a_{1}\right), \ldots, g_{N}\left(a_{1}\right) \in L_{r_{1}}$, where $L_{n}$ denotes the linear span of the vectors $e_{1}, \ldots, e_{n}$. Then one can take $a_{1}^{\prime}=e_{r_{1}+1}$, and we have $A_{1} \subset L_{r_{1}+1}$.

Now assume that $a_{1}, \ldots, a_{i-1}$ and $a_{1}^{\prime}, \ldots, a_{i-1}^{\prime}$ are already fixed, and $a_{1}, \ldots, a_{i-1} \subset L_{n}$ for some $n \in \mathbb{N}$. Then we can find $a_{i} \in L_{n}^{\perp}$ such that $g_{j}\left(a_{i}\right) \in L_{n}^{\perp}, j=1, \ldots, N$. Indeed, consider $g_{1}\left(e_{m}\right), m>n$. There are no more than $k$ non-zero coordinates among the first $n$ coordinates of $g_{1}\left(e_{m}\right)$ for each $m$. Then, as each line of the matrix of $g_{1}\left(e_{m}\right)$ cannot contain more than $k$ non-zero entries, the number of $m$ 's (i.e. the number of columns) for which there is a non-zero coordinate among the first $n$ coordinates of $g_{1}\left(e_{m}\right)$ is finite (not greater than $k n$ ). The same holds for each $j=1, \ldots, N$, so there is some $m$ such that the first $n$ coordinates of $g_{j}\left(e_{m}\right), j=1, \ldots, N$, are all zeroes.

The next step of homotopy in [7] is rotation of the two-dimensional subspace spanned by $g\left(a_{i}\right)$ and $a_{i}^{\prime}$, where $g \in \operatorname{Span}\left(g_{1}, \ldots, g_{N}\right)$, and then another rotation in the twodimensional subspace spanned by $a_{i}^{\prime}$ and $a_{i}$. Note that, although $\mathbb{B}^{(k)}(H)$ is not a linear space, we have $g \in G L^{(k N)}(H)$. Both rotations involve only finite number of coordinates, hence this part of the homotopy lies in $G L^{m}(H)$ for some $m \in \mathbb{N}$ and connects $f_{1}$ with $f_{2}$ such that $f_{2}(s) a_{i}=\left|f_{1}(s) a_{i}\right| a_{i}, s \in \mathbb{S}^{n}, i \in \mathbb{N}$.

Let $H^{\prime}=\operatorname{Span}\left(a_{1}, a_{2}, \ldots\right), H_{1}=\left(H^{\prime}\right)^{\perp}$. The next step of homotopy connects $f_{2}$ with $f_{3}$ such that $\left.\left(f_{3}\right)\right|_{H_{1}}=\left.\left(f_{2}\right)\right|_{H_{1}}$ and $\left.f_{3}(s)\right|_{H^{\prime}}=$ id. This homotopy only proportionally changes lengths of $f_{2}(s) a_{i}$, so does not change the number of non-zero entries. Let $p^{\prime}$ and $p_{1}$ denote the projections onto $H^{\prime}$ and $H_{1}$ respectively. Then $f_{3}(s)=p^{\prime}+f_{2}(s) p_{1}$. The next step of homotopy connects $f_{3}(s)$ with $f_{4}(s)=p^{\prime}+p_{1} f_{2}(s) p_{1}$, and does not increase the number of non-zero entries.

Recall that $H^{\prime}$ is spanned by an infinite set of the vectors from the basis, so we can write it as an infinite sum of infinitedimensional subspaces $H^{\prime}=H_{2} \oplus H_{3} \oplus \cdots$, each of which is spanned by an infinite set of the vectors of the basis. Then, with respect to the decomposition $H=H_{1} \oplus H_{2} \oplus H_{3} \oplus \cdots$, we can write $f_{3}(s)=\left(\begin{array}{cccc}u & & & \\ & & & \\ & & & \\ & & \ddots\end{array}\right)$ with $u$ having in each line and in each column mot more that $C$ non-zero entries for some $C$. The standard homotopy that connects $\left({ }^{u} u^{-1}\right)$ with $\left({ }^{1}{ }_{1}\right)$ (applied block-diagonal-wise) increases the number of non-zero entries in each line and in each column only twice, so the last step of homotopy that connects $f_{4}$ with $f_{5}$, where $f_{5}(s)=1$, also lies within $G L_{f}(H)$.

Corollary 6.2. $K_{0}\left(\mathbb{B}_{f}(H)\right)=K_{1}\left(\mathbb{B}_{f}(H)\right)=0$. In particular, the class of the projection from Proposition 2.3 is trivial.

Proof. This follows from Lemma 1.5 and from the isomorphism $K_{i}(A) \cong \pi_{i \pm 1}\left(G L_{\infty}(A)\right)$, $i \in \mathbb{Z} / 2$, [13], where $A$ is a $C^{*}$-algebra, $G L(A)$ denotes the group of invertibles in $A$, and $G L_{\infty}(A)=\lim _{n \rightarrow \infty} G L\left(M_{n}(A)\right)$. 


\section{MiscellaneA}

Lemma 7.1. The $C^{*}$-algebra $\mathbb{B}_{f}(H)$ has no polar decomposition, hence is not an $A W^{*}$ algebra.

Proof. Let $v \in \mathbb{B}(H)$ denote the isometry from Proposition 2.1. Let $h$ be the compact operator given by $h\left(e_{n}\right)=\lambda_{n} e_{n}$, with $\lambda_{n} \neq 0$ for any $n \in \mathbb{N}$ and $\lim _{n \rightarrow \infty} \lambda_{n}=0$. Set $a=v h$, where $v$ was defined in Proposition 2.1. Compactness of $a$ implies that $a \in \mathbb{B}_{f}(H)$, and Ker $h=0$ means that $v$ is determined in a unique way. As $v \notin \mathbb{B}_{f}(H)$, there is no polar decomposition within $\mathbb{B}_{f}(H)$. Therefore $\mathbb{B}_{f}(H)$ is not a Rickart algebra, hence not an $A W^{*}$-algebra [2].

Lemma 7.2. The stable topological rank of $\mathbb{B}_{f}(H)$ is $\infty$.

Proof. As in $\mathbb{B}(H)$, the unit projection 1 is the sum of the projections onto the subspaces spanned by odd and by even vectors of the basis, each of which is Murray-von Neumann equivalent to 1 , and both isometries $v_{1}$ and $v_{2}$ given by $v_{1}\left(e_{n}\right)=e_{2 n-1}, v_{2}\left(e_{n}\right)=e_{2 n}$, $n \in \mathbb{N}$, lie in $\mathbb{B}_{f}(H)$, hence $\mathbb{B}_{f}(H)$ is properly infinite, hence has infinite stable topological rank 9 .

Problem 7.3. Calculate the real rank of $\mathbb{B}_{f}(H)$.

Acknowledgement. The author is grateful to E. Troitsky for fruitful discussions.

\section{REFERENCES}

[1] B. Abadie, G. Cortiñas. Homotopy invariance through small stabilizations. J. Homotopy Relat. Struct. 10 (2015), 459-493.

[2] P. Ara. Left and right projections are equivalent in Rickart $C^{*}$-algebras. J. Algebra 120 (1989), 433-448.

[3] N. P. Brown, N. Ozawa. $C^{*}$-algebras and Finite-dimensional Approximations. Amer. Math. Soc., 2008.

[4] J. W. Calkin. Two-sided ideals and congruences in the ring of bounded operators in Hilbert space. Ann. Math. 42 (1941), 839-873.

[5] G. Cortiñas. Cyclic homology, tight crossed products, and small stabilizations. J. Noncommut. Geom. 8 (2014), 1191-1223.

[6] N. Higson, V. Lafforgue, G. Skandalis. Counterexamples to the Baum-Connes conjecture. Geom. Funct. Anal. 12 (2002), 330-354.

[7] N. Kuiper. The homotopy type of the unitary group of Hilbert space. Topology 3 (1965), 19-30.

[8] A. Lubotzky. Discrete groups, expanding graphs and invariant measures. Birkhäuser, 1994.

[9] M. A. Rieffel. Dimension and stable rank in the $K$-theory of $C^{*}$-algebras. Proc. London Math. Soc. 46 (1983), 301-333.

[10] J. Roe. Lectures on Coarse Geometry. University Lecture Series. Amer. Math. Soc., 31, 2003.

[11] J. Roe, R. Willett. Ghostbusting and property A. J. Funct. Anal. 266 (2014), 1674-1684.

[12] B. Tanbay. Pure state extensions and compressibility of the $l_{1}$-algebra. Proc. Amer. Math. Soc. 113 (1991), 707-713.

[13] R. Wood. Banach algebras and Bott periodicity. Topology 4 (1966), 371-389.

Moscow State University, Leninskie Gory 1, Moscow, 119991, Russia

E-mail address: manuilov@mech.math.msu.su 